\title{
LA INTERVENCIÓN EDUCATIVA ANTE UNA NUEVA DIVERSIDAD, ALTAS CAPACIDADES CON INGENIERÍA GENÉTICA
}

\author{
Maricela Zúñiga Rodríguez \\ Universidad Autónoma del Estado de Hidalgo, México. \\ innomary@hotmail.com
}

Fecha de Recepción: 18 Enero 2019

Fecha de Admisión: 30 Abril 2019

\section{RESUMEN}

México es uno de los países líderes en investigación e innovación científica en reproducción humana asistida aumentando el número de Clínicas de Reproducción Asistida. En 1986, sólo existían 2 clínicas particulares en todo el territorio, y actualmente existen alrededor de 70 incorporándose también clínicas del sector salud como un derecho a la reproducción humana. Por reproducción asistida se entiende al conjunto de método de médico-quirúrgicos, cuyo objeto es lograr la fecundación de un ser humano de manera diferente a las condiciones naturales. Con ello se han abaratado los costes de la reproducción asistida, siendo un 30\% y un 50\% más baratos en México con respecto a países europeos o Estados Unidos e incluso gratuitos en el sector salud como las clínicas del ISSSTE, del SSA o la Clínica de Nutrición, entre otras. Esto ha provocado que sean cada vez más nacimientos asistidos por ingeniería genética.

Ante el derecho a la reproducción asistida como un reciente hecho poblacional, en México, estos niños y niñas llegan a las aulas con la firme idea de los padres de que sus hijos son niños excepcionales o con altas capacidades con respecto al grupo escolar al que pertenecen, surgiendo las siguientes interrogantes: ¿Cómo se está transformando la población educativa presente en las aulas, ante el aumento de la ingeniería genética?; ¿Cuáles son las características psicológicas y cognitivas presentes en los alumnos considerando las capacidades establecidos en las aulas?

Es un estudio de enfoque mixto a partir de documentar casos atendidos en los Servicios de Educación Especial de la Secretaría de Educación Pública, de los resultados de su evaluación psicopedagógica se identifican sus características educativas, psicológicas y cognitivas, se muestran los resultados y las conclusiones obtenidas.

Palabras claves: altas capacidades; ingeniería genética; evaluación psicopedagógica

\section{ABSTRACT}

Mexico is one of the leading countries in research and scientific innovation in assisted human reproduction. Increasing the number of Assisted Reproduction Clinics. In 1986, there were 


\section{LA INTERVENCIÓN EDUCATIVA ANTE UNA NUEVA DIVERSIDAD, ALTAS CAPACIDADES CON INGENIERÍA GENÉTICA}

only 2 private clinics in the whole territory, and there are currently around 70 clinics in the health sector as a right to human reproduction. By assisted reproduction is meant the whole method of medical-surgical, whose purpose is to achieve the fertilization of a human being in a different way to natural conditions. This has reduced the costs of assisted reproduction, being $30 \%$ and $50 \%$ cheaper in Mexico with respect to European countries or the United States and even free in the health sector such as the ISSSTE clinics, the SSA or the Clinic of Nutrition, among others. This has led to more and more births assisted by this means.

Faced with the growing right to assisted reproduction as a recent population event, in Mexico, these children arrive in the classrooms with the firm idea of parents that their children are exceptional children or with high abilities with respect to the school group to which they belong, arising the following questions: How is the educational population present in the classrooms transforming before the increase in genetic engineering? What are the psychological and cognitive characteristics present in the students considering the capacities established in the classrooms?

It is a mixed approach study from documenting cases attended in the Special Education Services of the Ministry of Public Education, from the results of their psychopedagogical evaluation their educational, psychological and cognitive characteristics are identified, the results and the conclusions obtained are shown.

Keywords: high capacities; genetic engineering; psychopedagogical evaluation

\section{ÁREA TEMÁTICA:}

2. Educación, Psicología, historia y futuro.

2.3 Profesiones educativas y responsabilidad social. Intervención educativa y sus respuestas. Atención a la diversidad y dificultades de aprendizaje. Altas capacidades.

\section{ANTECEDENTES}

De acuerdo a Mcgee, (2003, las técnicas de reproducción asistida comenzaron a aplicarse en 1765 el alemán Ludwiy Jascobi quien las utilizó en salmones, y a finales del siglo XIX, se practicaron con un mamífero, una perra de la que nacieron tres crías. Afirman que en el siglo XV, se intentó -sin éxito- inseminar artificialmente a Juana de Portugal con semen de Enrique IV de Castilla. En 1978 nació la inglesa Luisa Brown, el primer humano fruto de una fecundación artificial in vitro; con lo que inicio la era de los llamados "bebé probeta". A partir del nacimiento de Luisa Brown han nacido cinco millones de personas por medio de la fecundación asistida. Existe una clasificación de las nuevas formas de fecundación en la ingeniería genética, algunas de ellas son:

Inseminación artificial

Fecundación in vitro

Maternidad subrogada

Reproducción asexual (clonación)

Dentro de las técnicas de procreación y reproducción asistida deben de valorarse la viabilidad ética y humana (esto es enaltezcan al ser humano y no lo conviertan en objeto) deben contemplarse en todo caso dos elementos:

La vida humana comienza con la concepción (genéticamente hablando madre e hijo son diferentes desde la unión de los gametos).

La dignidad de la sexualidad humana como medio natural de reproducción.

Ante estos nuevos procesos de procreación humana surge la siguiente interrogante:

¿Cuáles son las características psicológicas y cognitivas presentes en los alumnos considerando los rangos establecidos en las aulas? 
En los Servicios de Educación Especial que se ofrecen en la Secretaría de Educación Pública de Hidalgo, México, es frecuente que los docentes soliciten apoyo en casos de alumnos que presentan ansiedad, problemas de aprendizaje 0 altas capacidades, al llevar a cabo una evaluación psicopedagógica de éstos casos se ha podido detectar en la última década que son varios casos de alumnos nacidos por medio de inseminación o reproducción asistida. Lo que origina el interés por conocer mejor sus características personales, psicológicas y pedagógicas en relación con su escolaridad (Pérez, 2012).

Los bebés-probeta, esto es, los niños nacidos de fertilización in vitro, son más inteligentes y superiores en muchos aspectos que los engendrados de forma natural, según ha declarado el doctor Carl Wood, jefe del departamento de Obstetricia y Ginecología de la Universidad Monash en Melbourne, considerado como el más avanzado del mundo en esta técnica de fertilización artificial. De acuerdo con el citado profesor, un estudio realizado entre 25 niños engendrados por inseminación en probeta, de uno a tres años de edad, ha demostrado que su inteligencia, coordinación motriz y desarrollo físico son superiores a la media. Estos niños son también más sociables y más decididos a conseguir sus propios objetivos que los demás. El doctor Wood declaró ante el Colegio Australasiático de cirujanos que estas diferencias tienen probablemente un origen genético, por cuanto los padres de tales niños suelen ser luchadores y decididos y han sobrepasado muchas dificultades para tener hijos. El nivel socioeconómico de las familias implicadas es también superior al medio, ya que cada una de ellas ha tenido que pagar al menos 700 dólares de los 2.250 que cuesta el tratamiento, (Nota periodística de Cusi, 1984).

Ante el cada vez más derecho a la reproducción asistida como un reciente hecho poblacional, en países como México, estos niños y niñas llegan a las aulas con la firme idea de los padres de que sus hijos son niños excepcionales o con capacidades superiores al grupo escolar al que pertenecen.

Contando con los antecedentes analizados se procede a realizar un estudio sobre si la ingeniería genética favorece que existan personas con altas capacidades.

\section{OBJETIVO DE LA INVESTIGACIÓN}

Identificar en una alumna nacida con reproducción asistida sus características cognitivas y sociales dentro de un rango del grupo escolar al que pertenece.

\section{METODOLOGÍA}

\section{Participantes}

El estudio inició con 6 alumnos 2 del sexo masculino y 4 del sexo femenino de educación primaria en los rangos de edad de 6 a 11 años atenidos en la Unidad de Servicios de Apoyo a la Educación Regular (USAER) de Educación Especial, alumnos que en una primera fase fueron identificados por sus padres y maestros como potencialmente sobresalientes, por ello la siguiente fase fue una evaluación psicopedagógica, de las cuales se toma un caso ilustrativo mostrando los resultados de los dos más importantes instrumentos que se emplearon.

\section{Instrumentos}

El presente es un estudio con enfoque mixto cuantitativo y cualitativo, a partir de la evaluación psicopedagógica que realiza el equipo multidisciplinar de la USAER, la cual emplea distintos instrumentos como son:

a) Instrumentos no formales: Inventario de Aptitudes Sobresalientes, Nominación libre del profesor, portafolio de evidencias, instrumento ¿Adivina quién es?

b) Instrumentos formales: aplicados por equipo Multidisciplinar de la USAER: Psicólogo, tra- 


\section{LA INTERVENCIÓN EDUCATIVA ANTE UNA NUEVA DIVERSIDAD, ALTAS CAPACIDADES CON INGENIERÍA GENÉTICA}

bajadora social, pedadogogo, los instrumentos empleados son WISC IV; BAS 1, 2, 3, Pruebas de creatividad.

\section{Procedimiento}

Ficha de identificación

Nombre: María de la Concepción (El nombre es ficticio por respeto a la identidad de la niña)

Género: Femenino

Fecha de nacimiento: 14 de febrero del 2007

Edad: 7 años

Escolaridad: $2^{\circ}$ de primaria

Fecha de estudio: 1 de agosto del 2018

\section{RESULTADOS A PARTIR DE ESCALA WISC IV Y BAS 1 Y 2}

\section{Resultados de Batería de Socialización (BAS, 1 y 2) versión para profesores y padres}

La Batería de Socialización (BAS) es un conjunto de escalas de estimación para evaluar la socialización de niños y adolescentes en ambientes escolares y extraescolares. Se aplicó la versión para profesores (BAS-1) y padres (BAS-2) en distintas fechas, durante los meses de junio y julio del 2018. El objetivo de este instrumento era estimar cuatro dimensiones facilitadoras de la socialización y tres dimensiones perturbadoras, así como una escala global de adaptación social o Criterialsocialización.

\section{Resultados de BAS 1 dirigido a profesores}

La BAS 1 se aplicó a dos profesores de la menor, los resultados obtenidos de las cuatro dimensiones facilitadoras son los siguientes:

Liderazgo (Li): no muestra liderazgo lo que se traduce en falta de popularidad, falta de iniciativa y desconfianza en sí misma.

Jovialidad (Jv): se muestra introvertida, con dificultad para mantener relaciones sociales, lo que dificulta su integración.

Sensibilidad social (Ss): Ios resultados son bajos al evaluar que existe una despreocupación hacia aquellos que pudieran tener problemas.

Respeto-autocontrol (Ra): tiene la capacidad de acatar reglas y normas, aunque se le tiene que insistir en ellas para facilitar la convivencia.

En las tres dimensiones perturbadoras son los siguientes:

Agresividad-terquedad (At): muestra aspectos altos de conducta impositiva, perturbadora y antisocial. Su principal característica es la resistencia a las normas, la indisciplina y con altos índices de agresión, que se relaciona con la hiperactividad motriz.

Apatía- retraimiento $(\mathrm{Ar})$ : la refleja como introvertida con tendencia al aislamiento. Puede manifestar falta de energía y pobre iniciativa ante las actividades.

Ansiedad-timidez (An): no muestra miedos ni nerviosismos ni timidez.

Con respecto a la apreciación global de la socialización o Criterial-socialización (Cs) que tienen los profesores de la menor, sugiere que la menor presenta deficiente adaptación social, principalmente en la manera de entablar relaciones sociales con otros niños de su edad e incluso adultos. Los resultados sugieren retraimiento social hasta la posibilidad de introversión y aislamiento.

\section{Resultados de BAS 2 dirigido a padres}

La BAS 2 se aplicó la madre y el padre de la menor, los resultados obtenidos de las cuatro dimensiones facilitadoras son los siguientes: 
Liderazgo (Li): en el hogar muestra liderazgo, confianza en sí misma e iniciativa.

Jovialidad (Jv): Ios resultados sugieren que la menor puede mostrarse introvertida, pero es probable que con otros miembros de la familia muestre sociabilidad.

Sensibilidad social (Ss): expresa despreocupación hacia aquellos que pudieran tener problemas.

Respeto-autocontrol $(\mathrm{Ra})$ : tiene dificultades para acatar reglas y normas establecidas en el círculo familiar, teniéndole que insistir en ellas para facilitar la convivencia.

En las tres dimensiones perturbadoras son los siguientes:

Agresividad-terquedad (At): manifiesta en algunas ocasiones conductas impositivas, mostrando resistencia a las normas, mostrando actitudes de indisciplina.

Apatía- retraimiento (Ar): la identifican como introvertida con tendencia al aislamiento. Puede manifestar falta de energía y pobre iniciativa ante las actividades.

Ansiedad-timidez (An): no muestra miedos ni nerviosismos ni timidez.

Con respecto a la apreciación global de la socialización o Criterial-socialización (Cs) que tienen los padres de la menor, existe una dualidad en los resultados debido, probablemente al tiempo de convivencia. Los resultados sugieren que la menor puede presentar problemas de socialización con otros miembros de su familia o con personas externas a su círculo familiar.

\section{Resultados de la Escala Wechsler de Inteligencia para Niños-IV}

Ante la evaluación la alumna, mostró en sus inicios disposición, anteponiendo que si la actividad consistía en escribir ella no lo realizaría porque eso le causa incomodidad. Se le cuestionó al respecto, a lo que respondió que no le gusta nada que trate sobre la escritura.

Para la aplicación se le dieron cuatro consignas, en forma de reglas que permitirían la aplicación de la escala: 1) realizar las actividades que se le solicitaran; 2) manifestar dudas sobre las actividades antes de realizarlas; 3) no tocar ningún material (incluyendo maletín, hojas, lápices, entre otros) si no son requeridos en la actividad; y 4) no salir del espacio destinado para la aplicación, hasta concluir 0 cuando se le pida.

No tuvo problemas con la consigna uno y dos, pero al respecto de la tres y la cuatro, no respetó lo acordado, ya que en reiteradas ocasiones tomaba objetos que no estaban dentro de las actividades, poniendo en riesgo su atención y concentración ante las actividades solicitadas. En algún momento quiso salir a buscar a su familia sin consultar antes, entonces se le tuvo que recordar las cuatro reglas manifestadas al principio, y de las cuales ella tenía conocimiento. Dicha actitud sugiere falta de límites y pobre atención a consignas dadas.

En reiteradas ocasiones mencionaba que las actividades le cansaban, pero éstas no podían interrumpirse porque no alcanzaba los protocolos prestablecidos de la prueba para ser canceladas, por tanto se optó por realizar ejercicio de relajación y flexibilidad mental entre actividades para poder continuar. Se le permitió no permanecer sentada en un solo lugar para evitar la fatiga cognitiva.

Al final de la prueba, mostró disposición y cambio de actitud, se le cuestionó sobre su estado de ánimo, mencionando que se encontraba bien y que quería reunirse con sus familiares.

\section{Resumen de puntuaciones}

Los resultados de la Escala Wechsler de Inteligencia para niños IV (WISC-IV), brindan información importante acerca del funcionamiento cognitivo del niño, además provee información cuantitativa y cualitativa que es posible interpretar mejor junto con una historia clínica, observaciones psicopedagógicas y otro tipo de evaluaciones clínicas. A continuación se presenta el resumen de puntuaciones obtenidas por la menor: 


\section{LA INTERVENCIÓN EDUCATIVA ANTE UNA NUEVA DIVERSIDAD, ALTAS CAPACIDADES CON INGENIERÍA GENÉTICA}

(Cuadro 1. Elaboración propia)

\begin{tabular}{|l|c|c|l|}
\hline Escalas & $\begin{array}{c}\text { Índice } \\
\text { compuesto }\end{array}$ & $\begin{array}{c}\text { Intervalo de } \\
\text { confianza de } \\
95 \%\end{array}$ & Categoría \\
\hline Comprensión Verbal (CV) & 146 & $136-150$ & $\begin{array}{l}\text { Promedio alto/Punto fuerte } \\
\text { normativo }\end{array}$ \\
\hline Semejanzas & 18 & & \\
\hline Vocabulario & 10 & & \\
\hline Comprensión & 12 & & \\
\hline (Información) & 13 & & \\
\hline $\begin{array}{l}\text { Razonamiento Perceptual } \\
\text { (RP) }\end{array}$ & 112 & $103-119$ & Promedio/Dentro de límites \\
\hline Cubos & 11 & & \\
\hline Conceptos & 6 & & \\
\hline Matrices & 13 & & \\
\hline (Figuras incompletas) & 6 & & \\
\hline Memoria de Trabajo (MT) & 120 & $111-127$ & Promedio/Dentro de límites \\
\hline Dígitos & 11 & & \\
\hline Letras y números & 6 & & \\
\hline (Aritmética) & 121 & $110-127$ & Promedio/Dentro de límites \\
\hline $\begin{array}{l}\text { Velocidad } \\
\text { Procesamiento (VP) }\end{array}$ & 8 & & \\
\hline Claves & 9 & & \\
\hline Búsqueda de símbolos & 10 & & \\
\hline (Animales) & 106 & $101-111$ & Promedio/Dentro de límites \\
\hline CI Total & & & \\
\hline
\end{tabular}

\section{Capacidad intelectual total}

La Capacidad Intelectual Total o Cl Total, es la puntuación más confiable que se obtiene de la Escala Wechsler de Inteligencia para niños IV. Es la capacidad global y agregada presente como un todo, en la que intervienen factores sociales, cognitivos, personales y ambientales. En el caso de niña evaluada, se obtuvo un $\mathrm{Cl}$ Total de 106. Su capacidad intelectual se clasifica como Promedio/Dentro de límites, en una media estandarizada de la capacidad intelectual. Existe alta probabilidad (95\%) de que su verdadero $\mathrm{Cl}$ Total se situé entre los valores101-111.

La Cl Total de la menor se sitúa en el percentil 66, es decir que su puntuación mejora al $66 \%$ de las personas de su edad en la muestra estándar de población. En la comparación entre el índice compuesto más alto (146) y el más bajo (112), la diferencia es alta (34), por tanto el Cl Total de la menor debe interpretarse con cautela.

\section{Interpretación de los índices del CI Total}

\section{Índice de Comprensión Verbal (ICV)}

Es una medida de la formación de conceptos verbales, razonamiento verbal y conocimiento adquirido del propio ambiente. Con las subpruebas que lo componen, se puede identificar cómo la menor comunica conocimientos y razona utilizando las experiencias o procedimientos previamente aprendidos (inteligencia cristalizada). El valor obtenido por la niña es de 146 en el rango percentil de 99.9 en el intervalo de confianza (al 95\%) de 136-150, que clasifica a la menor como Promedio 
alto/Punto fuerte normativo. La puntuación obtenida por la menor, se encuentra $+3 \mathrm{DE}$ respecto a la media, siendo equivalente al rango percentil arriba mencionado.

EI ICV se desarrolla en función de las oportunidades y experiencias educativas formales e informales y es una medida de la inteligencia cristalizada. Se obtiene de la evaluación de tareas que exigen definir palabras, expresar similitudes conceptuales entre palabras o responde a cuestiones que implican conocimiento de principios generales y situaciones sociales. En el caso de los resultados de las subpruebas aplicadas a la menor, la diferencia entre la puntuación escalar más alta y la más baja es de 8 puntos, siendo alta y sugiere que el resultado obtenido debe ser analizado con cautela, lo cual indica que su capacidad intelectual no se puede resumir con un valor unitario, por lo que la interpretación de este índice deberá realizarse con cautela.

La capacidad de comprensión verbal de la menor se considera un punto fuerte significativo cuando se compara con personas de su edad en la muestra estándar de población. En realidad la diferencia entre esta capacidad (ICV) y sus aptitudes en otras áreas es tan grande que no es frecuente observarla en otros casos de su edad. Por tanto la capacidad de comprensión verbal de la menor es un punto fuerte personal notable, y una entidad notable para una intervención educativa para potenciar su desarrollo.

\section{Índice de Razonamiento Perceptual (IRP)}

Es una medida del razonamiento fluido, el procesamiento espacial y la integración visomotora. Esta capacidad se evalúa mediante diversas tareas como reproducir un modelo construido con cubos, seleccionar el dibujo de un grupo que no forma parte del mismo o complementar una matriz. En el caso de la menor, la comparación y diferenciación de los resultados, no es grande y sugiere que el resultado en el IRP es una buena medida para medir el razonamiento perceptivo de la menor.

El valor obtenido por la niña es de 112 puntos, se sitúa en el rango percentil 79, en el intervalo de confianza al (95\%) 103-119, que la clasifica como Promedio/Dentro de límites. Los datos sugieren que la capacidad de razonamiento perceptual se encuentra en promedio dentro de la media estandarizada. Al analizar los resultados en cada subprueba que compone el IRP, existe una variabilidad de los resultados, ya que la diferencia es grande (7), es decir, su amplitud es mayor de 5 puntos, lo cual indica que su capacidad intelectual general no se puede resumir con un valor unitario.

\section{Índice de Memoria de Trabajo (IMT)}

Este índice provee una medida de las capacidades de memoria de trabajo de la menor. Las tareas que requieren memoria de trabajo demandan la capacidad de conservar temporalmente la información dentro de la memoria, ejecutar alguna operación o manipulación con ella y producir un resultado. La memoria de trabajo implica atención, concentración, control mental y razonamiento. Se evalúa mediante tareas que exigen repetir una lista de cifras o recordar series de números y letras en un orden determinado.

El valor obtenido por la menor es 120 puntos en rango percentil 91, en el intervalo de confianza (al 95\%) 111-126 que la sitúa en Promedio/Dentro de límites. El resultado del IMT es una medida de la memoria a corto plazo y refleja la capacidad para retener temporalmente en la memoria cierta información, trabajar u operar con ella y generar un resultado. Se han comparado las puntuaciones que componen al índice, siendo no tan grande la diferencia (menos de 5 puntos) lo que sugiere que el resultado es una buena medida de la memoria de trabajo de la menor.

\section{Índice de Velocidad de Procesamiento (IVP)}

Existe una correlación significativa entre la velocidad de procesamiento y la capacidad cognitiva general, el IVP da una medida de la capacidad del niño para rastrear, secuenciar o discriminar de 


\section{LA INTERVENCIÓN EDUCATIVA ANTE UNA NUEVA DIVERSIDAD, ALTAS CAPACIDADES CON INGENIERÍA GENÉTICA}

manera rápida y correcta la información visual simple. En el caso, obtuvo 121 puntos, en un rango percentil 92, en el intervalo de confianza (al 95\%) de 110-127, que la sitúa en Promedio/Dentro de límites. Es una medida de velocidad de proceso y representa la capacidad de la menor para realizar tareas simples, de tipo administrativo. Esta capacidad se evalúa mediante tareas que requieren copiar rápidamente símbolos que están emparejados con números según una clave 0 identificar la presencia 0 ausencia de un símbolo modelo de una fila de símbolos. Al comparar las puntuaciones, la diferencia de sus puntuaciones no es grande (llega a 5) y sugiere que el resultado del índice es una buena medida de su velocidad de procesamiento.

\section{DISCUSIÓN}

\section{Intervención educativa}

El primer instrumento permitió identificar aspectos de la conducta social de la menor, a través de la Batería de Socialización aplicada en dos versiones: BAS-1 para profesores y BAS-2 para padres. Su aplicación fue de manera individual y la finalidad fue detectar los factores que facilitan u obstaculizan la socialización en ambientes escolares y familiares. Se ha identificado que en ambos instrumentos los resultados apuntan a puntuaciones bajas en los factores facilitadores y altas en los factores perturbadores, que da pauta para identificar que la menor muestra dificultades para una integración social en ambos contextos, lo que le propicia problemas de conducta, que por la edad de la menor, están en la oportunidad de ser atendidas.

De acuerdo con los resultados obtenidos de la aplicación del WISC-IV para niños, la menor de 7 años, obtuvo una Capacidad Intelectual Total de 106, que la clasifica como Promedio/Dentro de límites, con alta probabilidad (95\%) de que su verdadero $\mathrm{Cl}$ Total se situé entre los valores 101-111. Los resultados obtenidos, la sitúan en el promedio cuando se compara con otras personas de su edad en la muestra estándar de la población, y que viene a dar respuesta a las preguntas y objetivos iniciales del presente este estudio.

Con respecto a los resultados obtenidos en cada índice compuesto (ICV, IRP, IMT, IVP), es notorio un alza en el Índice Comprensión Verbal (ICV) siendo de 146, en intervalo de confianza al 95\%, posicionándola en Promedio alto/Punto fuerte normativo, en comparación con la muestra estándar de la población. Dicha puntuación obtenida por la menor, se encuentra +3 respecto a la media, siendo equivalente al rango percentil 136-150. Los resultados muestran desequilibrio con respecto a los otros índices, al poner en evidencia que la menor ha desarrollado más las habilidades para comunicar conocimientos utilizando las experiencias o procedimientos previamente aprendidos en su crianza.

Esta situación la pone en desventaja en los espacios áulicos y en las actividades planeadas por sus docentes para promover aprendizajes de otra índole (que requieran el uso de otras habilidades). Entonces, la menor se muestra selectiva ante aquellas actividades en las cuales conoce previamente y puede manejar, anteponiendo negativa a otras que puedan ayudarle a desarrollar nuevas habilidades.

Se recomienda a los padres que la menor desarrolle hábitos que le permitan fomentar otras habilidades necesarias, no solo para los espacios áulicos, sino para mejorar su desarrollo social, cognitivo y personal; atendiendo a sus necesidades educativas. No es recomendable centrarse solo en aquellas actividades que la menor quiere realizar, es necesario promover, a través de la curiosidad o el asombro, otro tipo como la escritura, el dibujo, la pintura, los ejercicios al aire libre, entre otros, que promuevan diversas habilidades. La finalidad es que, desarrolle su Capacidad Intelectual de manera equilibrada y constante. 
Los padres de familia tomen acuerdos sobre su figura de autoridad que mostrarán ante la menor, para no caer en doble discurso, que genere confusión sobre las reglas y normas que la familia debe tener para una mejor convivencia. La misma recomendación será para la escuela, donde la familia deberá respetar el cumplimiento de las normas y sanciones que la escuela establece, sin discutirlas o demeritarlas frente a la menor.

Se solicita el manejo y el trabajo de la inteligencia emocional así como, el desarrollo de habilidades de socialización, en diferentes contextos. Realizar actividades en familia que impliquen ejercicios, juegos de mesa tanto en casa como en la escuela, todo ello para el aprendizaje y manejo de normas y reglas, Sánchez, V, Sánchez Reza, M. (2014).

La negociación entre la escuela y los padres de familia es fundamental para mejorar la convivencia y las condiciones que faciliten el desarrollo de la menor. Evitar barreras entre profesores y padres de familia para que exista coherencia entre lo que se dice en la casa y en la escuela. Evitar barreras por parte de los profesores hacia la menor con respecto a su historia personal y sus características.

\section{CONCLUSIONES}

A medida que las nuevas técnicas de reproducción asistida hacen posibles a nuevas poblaciones, habrá que hacerles frente a los retos que implican como son estigmas, prejuicios y falsos positivos de capacidades superiores, al ser el genoma humano que aporta la información genética sobre las características físicas y cognitivas de los niños y niñas, con la reproducción asistida no se garantiza que los niños sean personas con altas capacidades como en los seis casos estudiados donde sus resultados son de personas dentro del rango normal.

Los resultados de los dos instrumentos muestran que es una alumna dentro de los parámetros de la normalidad y que los características que la hacen sobresalir son sus conductas que trascienden las reglas, establecidas en la escuela, razón para que los padres consideran que no les brinda la atención necesaria a sus necesidades educativas las cuales consideran extraordinarias y que los instrumentos dan prueba que son niños como cualquier otro en donde se ve comprometida las prácticas de crianza y sobre proteccionismo originadas por los padres, con mayor énfasis en la madre. Esperando que sus prácticas de crianza también las realicen los docentes de sus hijos, que originan conflictos y conductas disruptivas que buscan solución en Educación Especial.

\section{REFERENCIAS BIBLIOGRÁFICAS}

Cusi, F. (1984) Los "bebés-probeta" son más inteligentes que los demás, según el doctor australiano Wood El País. España recuperado en: https://elpais.com > Sociedad

Glenn, Mcgee. (2003). El bebé perfecto. Tener hijos en el nuevo mundo de la clonación y la genética. España: Gedisa.

Pérez, M. Et. Al. (2012). El plan andaluz de atención a las altas capacidades intelectuales. Revista INFAD 2012, №2, Volumen 1, pp. 201-208.

Mukherjee, Siddhartha. (2016). The Gene. An intimate History. USA: Scribner.

SEP (2006) Propuesta de Intervención: Atención educativa a alumnos y alumnas con aptitudes sobresalientes. México: SEP.

SEP, (2000). La integración Educativa en el aula regular. Principios finalidades y estrategias. SEP. Materiales de apoyo. México: Recuperado de: https://www.educacionespecial.sep.gob.mx/.../2Integracion Educativa aula regular.pdf

Silva Moreno F. y M. C. Martorell, Pallás. (2018). Batería de Socialización BAS 1 y 2 para profesores y padres. Manual. Madrid: TEA. 


\section{LA INTERVENCIÓN EDUCATIVA ANTE UNA NUEVA DIVERSIDAD, ALTAS CAPACIDADES CON INGENIERÍA GENÉTICA}

Sánchez, V, y Sánchez Reza, M. (2014). Inteligencias múltiples y capacidad superior: perfil e intervención. Revista INFAD 2014, №1, Volumen 7, pp. 591.

Weshler, David. (2007). WISC-IV. Escala Wechsler de Inteligencia para niños-IV. Manual Técnico. Versión estandarizada. Manual Moderno: México. 